\title{
Información sobre internacionalización de empresas de Castilla-León
}

\author{
Por María-Valle Santos-Álvarez y María-Teresa García-Merino
}

\begin{abstract}
Resumen: La decisión empresarial de internacionalización está relacionada con el aprovechamiento de las oportunidades que proporcionan los mercados exteriores y por lo tanto depende del acceso a la información y del esquema cognitivo del decisor empresarial, el directivo. En este estudio se analiza cuáles son las fuentes de información que proporcionan los estímulos informativos que convergen en la decisión de internacionalización y la influencia que ejercen las propiedades cognitivas de los directivos. Para ello se realizó una encuesta entre empresas castellano-leonesas en dos sectores: "tecnologías de la información" y "piedra natural". Los estímulos informativos se desglosan en tres conceptos (atractivos, barreras y apoyos) y se reconocen distintas fuentes de información (Icex, Excal, Cámaras de Comercio y asociación sectorial). Las propiedades cognitivas se refieren a proactividad y nivel de conocimiento. Los resultados revelan la importancia relativa de las diferentes fuentes de información consideradas. Además reflejan cómo la información se combina con las propiedades cognitivas de los directivos para conformar la valoración de los factores que convergen en la internacionalización.

Palabras clave: Información, Internacionalización, Proactividad, Decisiones, Empresas, Directivos, Castilla y León, España.

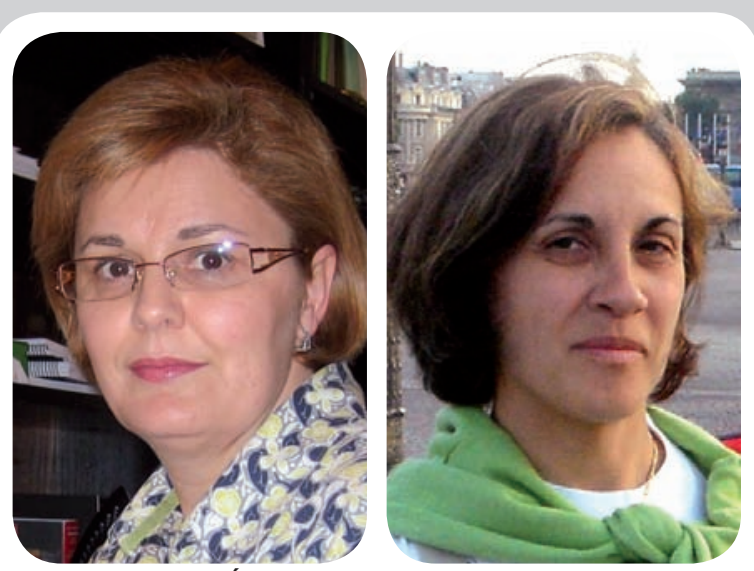

Mㅁ Valle Santos Álvarez y Mํ Teresa García Merino, doctoras en ciencias económicas y empresariales por la Universidad de Valladolid, son ambas profesoras titulares de universidad. Su investigación se centra en el ámbito de la estrategia empresarial, especialmente en: el estado de la investigación en dirección estratégica, procesos de creación de empresas, cambio estratégico, internacionalización y sesgos cognitivos. Son autoras de numerosos artículos de revista y comunicaciones en congresos, tanto nacionales como internacionales. Participan en proyectos de investigación financiados por diversas entidades (Ministerio de Educación y Ciencia, y Junta de Castilla y León).
\end{abstract}

\section{Title: Information on internationalization in Castilla-Leon companies}

Abstract: Management decision to initiate or develop internationalization depends on the ability to take advantage of opportunities afforded by foreign markets. Factors determining whether these opportunities may be profited from are: access to information and the cognitive approach of the decision-making agent, namely the manager. In our research we explore which sources of information provide the stimuli impacting the decision to internationalize and assess the influence of management's cognitive approach. To achieve this we conducted a survey amongst Spanish firms in the region of Castilla and León in two specific areas: "information technologies" and "natural stone". Informative stimuli are split into three concepts (appealing factors, barriers and support) and we draw on several sources of information (Icex, Excal, Chambers of Commerce and trade associations). We address two cognitive areas: proactivity and level of knowledge. Findings highlight the relative importance of the different sources of information explored in the analysis. Our research also evidences how the information received by managers merges with their own cognitive approaches forming the basis to assess the factors impacting the decision to internationalize.

Keywords: Information, Internationalization, Proactivity, Decisions, Companies, Managers, Castilla y Leon region, Spain.

Santos-Álvarez, María-Valle; García-Merino, María-Teresa. "Información sobre internacionalización de empresas de Castilla-León”. El profesional de la información, 2009, julio-agosto, v. 18, n. 4, pp. 407-412.

DOI: 10.3145/epi.2009.jul.07

\section{Introducción}

LA INTERNACIONALIZACIÓN EMPRESARIAL es un fenómeno amplio en el que convergen diversos factores, lo que ha dado lugar a distintas líneas de investigación.
Se han revisado cuestiones como: el perfil de las empresas exportadoras, la decisión estratégica de entrada en los mercados exteriores, el conjunto de factores que influyen en el comportamiento internacional de las empresas, el entorno competitivo que favorece el desarrollo inter- nacional, los recursos organizativos más relevantes, etc.

Recientemente la investigación ha dirigido el análisis hacia la figura del directivo y su influencia en el proceso y la gestión de la internacionalización empresarial, estudiándose la influencia de variables de- 
mográficas como por ejemplo edad, sexo, formación, etc. Sin embargo la literatura ha reconocido que individuos con similares particularidades demográficas muestran diferencias en el proceso de internacionalización empresarial (Clarke; Mackaness, 2001; Manolova et al., 2002).

A partir de este punto la investigación tiene en cuenta la influencia del directivo desde una perspectiva cognitiva y los mecanismos mediante los cuales los individuos adquieren, transforman y usan la información (Matlin, 2002). Con esta perspectiva se examina la forma en que el directivo adquiere, almacena y usa la información en el proceso de internacionalización. Se ha destacado la importancia del acceso a las fuentes de información (Shane, 2002, 2003) y la importancia de la búsqueda de información en el desarrollo de la internacionalización (Garg et al., 2003:726).

En esta investigación nos centramos en el análisis de la información que los directivos utilizan en el proceso de internacionalización empresarial. En concreto, nos planteamos:

- cuáles son las fuentes de información de las que se nutren en las decisiones

- cómo afecta la actitud de los directivos ante la necesidad de información que requiere la internacionalización de la empresa.

La investigación se organiza en diferentes secciones. A continuación revisamos la importancia que el acceso a la información tiene en la internacionalización empresarial, lo que hace posible la formulación del modelo de análisis. Después presentamos su aplicación empírica a partir de un estudio preliminar. Posteriormente revelamos los resultados que se deducen de los análisis realizados. Finalmente recogemos las principales conclusiones que se desprenden de la investigación y destacamos sus implicaciones para futuras investigaciones.

\section{La información en el proceso de internacionalización}

Nos referimos a una serie de compromisos que establecen vínculos entre las empresas y los mercados exteriores (Alonso, 1994), desde las exportaciones hasta operaciones de inversión directa en el exterior pasando por alianzas estratégicas con socios extranjeros. Sin embargo, la exportación constituye, mayoritariamente, la fase inicial del proceso de internacionalización empresarial, sobre todo para pequeñas y medianas empresas.

\section{"La internacionalización abarca exportaciones, operaciones de inversión directa en el exterior e incluso alianzas estratégicas con socios extranjeros"}

En la internacionalización empresarial convergen factores del entorno competitivo y las particularidades de la empresa. No obstante, la decisión no emerge de forma natural por la simple combinación de esos factores sino por la intervención del directivo. No sólo son los factores objetivos los que intervienen en la toma de decisiones, sino también la percepción y valoración personal que el decisor empresarial se ha formado sobre ellos (Weick, 1979). El proceso de percepción se convierte así en el elemento central del análisis de las decisiones directivas, en general, y de la decisión de internacionalización en particular (Burton; Schlegelmilch, 1987; Manolova et al., 2002).

Esto nos lleva a plantearnos por qué algunos directivos son capaces de descubrir y explotar las oportunidades de los mercados exteriores y otros no. La respuesta la podemos encontrar en la literatura centrada en el reconocimiento de oportunidades empresariales (Gaglio; Katz, 2001; Shane; Venkataraman, 2000). Desde este enfoque la capacidad de descubrir oportunidades empresariales se atribuye a dos grupos de factores:

- información relevante sobre la oportunidad;

- propiedades cognitivas del individuo necesarias para explotarla.

\section{Acceso a información}

Se trata de que los estímulos informativos sean reconocidos y atendidos por los directivos. Esta es una cuestión importante (Ozgen; Baron, 2007) ya que frecuentemente se admite que muchos de los problemas que surgen en el proceso de internacionalización empresarial se deben a la ignorancia del directivo sobre las fuentes de información (Katsikeas; Morgan, 1994). Esa información versa sobre (Claver; Quer, 2001; Santos; García, 2008):

- El atractivo o valoración de la internacionalización como alternativa estratégica para aprovechar las oportunidades de negocio en los mercados exteriores (Ramírez; Rosell, 2006).

- Las barreras u obstáculos actitudinales, estructurales, operativos o de cualquier otro tipo que dificultan o impiden el deseo de la empresa de iniciar, implantar o mantener su proyecto internacional (Leonidou, 1995: 13).

- Los programas de ayuda que proporcionan diferentes organismos para facilitar la internacionalización de las empresas, que pueden ser (Calderón; Fayos, 2004): de información, consulta, promoción comercial (asistencia a ferias, misiones, prospección de mercados), iniciación a la exportación, servicios individualizados a empresas, formación, financiación y seguros, etc. 
La detección de oportunidades requiere que la información no sólo esté disponible, sino que además sea reconocida por quien tiene que tomar la decisión. El exceso de información, su ambigüedad y las limitaciones cognitivas individuales provocan que el decisor empresarial reconozca únicamente una parte de la información disponible (Baron, 1998). La investigación refleja que los criterios que rigen la selección de los estímulos son muy variados. En ocasiones se seleccionan según el emisor. Por ejemplo, estímulos prevenientes de agentes económicos o socios en los que confiamos tienen mayor probabilidad de ser atendidos (Carolis; Saparito, 2006; McEvily et al., 2003). Los organismos públicos, como emisores de mensajes, gozan de amplia legitimidad organizativa $\mathrm{y}$, por lo tanto, las señales informativas que emiten se reciben con mayor interés y atención (Dutton; Ashford, 1993; Dutton et al., 2001; Ling et al., 2005).

\section{"Para aprovechar las oportunidades, la información debe estar disponible y ser reconocida por quien toma las decisiones"}

\section{Propiedades cognitivas indivi- duales}

El segundo factor determinante del reconocimiento de oportunidades se refiere al esquema cognitivo $\mathrm{y}$, sobre todo, a la estructura cognitiva que asiste al directivo a estar atento a las mismas. Nos referimos a oportunidades halladas en un proceso de búsqueda deliberada e investigación fructífera por parte del empresario, no a las que surgen como consecuencia de la suerte o el descubrimiento accidental (Gaglio;

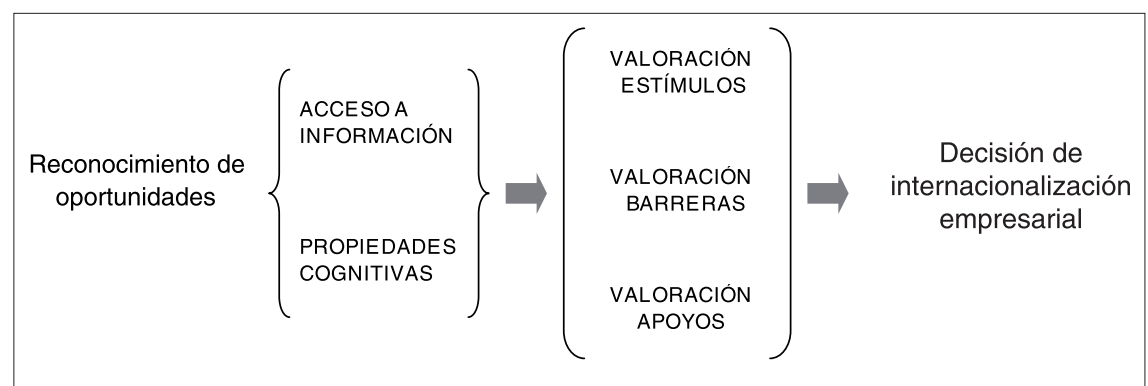

Figura 1. Modelo de análisis

Katz, 2001). Esa habilidad se ha denominado vigilancia (alertness) y está directamente relacionada con la cantidad de tiempo y esfuerzo que dedica el empresario a buscar y a seleccionar fuentes de información (Kaish; Gilad, 1991). El esquema cognitivo incluye también la actitud del individuo a correr riesgos y a poner en práctica iniciativas, lo que se conoce como "comportamiento proactivo": tener iniciativa para la mejora de las circunstancias actuales o para la creación de nuevas circunstancias (Crant, 2000).

\section{Aplicación empírica}

La aplicación empírica del estudio viene marcada por dos propósitos concretos:

- estudiar las fuentes de información y los emisores que los directivos castellano-leoneses toman como referencia para decidir internacionalizarse;

- evaluar la influencia de la información y la actitud individual de los directivos respecto a la valoración de los factores que determinan el proceso de internacionalización.

Elaboramos un cuestionario que fue remitido por correo postal a una muestra de empresas españolas de la región de Castilla y León (España) en dos sectores: tecnologías de la información y la comunicación (en adelante TIC) y piedra natural (en adelante PN). La selección de estos sectores responde a que ambos están entre los de mayor interés para el gobierno regional de Castilla y León a la hora de impulsar la internacionalización del tejido empresarial de la región -están incluidos en el II Plan de Internacionalización Empresarial de Castilla y León (2008-2011)-.

En el cuestionario se pedía a los directivos que indicaran su valoración sobre los elementos que intervienen en la decisión de internacionalización y el grado de información que reciben de distintos emisores. Los estímulos informativos se desglosan en las tres categorías citadas anteriormente: atractivo de la internacionalización para la empresa, barreras, y programas de ayuda que ponen en marcha distintos organismos.

Respecto a las fuentes de información nos centramos en los organismos comprometidos en la promoción de la internacionalización empresarial:

- Cámaras de comercio,

- Ade Internacional Excal, agencia de Castilla y León para la promoción de la internacionalización empresarial,

- Instituto Español de Comercio Exterior (Icex), organismo nacional dedicado al impulso de la internacionalización; y

- asociaciones sectoriales de empresarios (Aetical en el sector TIC y Pinacal en el sector PN).

Para cada factor que interviene en la internacionalización, preguntamos cómo valora el directivo el grado de información proporcionada por cada uno de los organismos considerados. Para todas las preguntas recogidas en el cuestionario respecto a los factores determinan- 
tes de la internacionalización y sobre las fuentes de información las respuestas se plantean en una escala Likert de 5 puntos.

En el cuestionario se incluyen también preguntas sobre la actitud del directivo en el proceso de recopilación de información:

- actitud más o menos proactiva del decisor empresarial;

- grado de conocimiento de distintos instrumentos y foros de promoción de la internacionalización de las empresas castellanoleonesas (cuadro 1).

Las empresas consideradas fueron las adscritas a la correspondiente asociación sectorial (Aetical y Pinacal) y además habían manifestado su interés por el proceso de internacionalización. En el sector de PN de las 53 empresas asociadas tan sólo 15 han mostrado interés por la internacionalización (Castilla y León Exporta, 2007. a). En el caso de las empresas del sector TIC, de las 162 empresas asociadas, las interesadas en la internacionalización son tan sólo 33 (Castilla y León Exporta, 2007.b). De los cuestionarios enviados recibimos 35 respuestas válidas, 21 del sector TIC y 14 del PN. Aunque el tamaño de la muestra es evidentemente muy reducido, debemos señalar que recoge mayoritariamente a las empresas de los dos sectores que están interesadas en la internacionalización. Por lo tanto, creemos que los resultados obtenidos sirven como ejemplo ilustrativo del uso de la información en el proceso de internacionalización empresarial.

\begin{tabular}{|l|l|}
\hline $\begin{array}{l}\text { ¿Conoce los seminarios y cursos de exportación promovidos } \\
\text { por Icex y las cámaras de comercio? }\end{array}$ & $\square \mathrm{Si} \square \mathrm{No}$ \\
\hline ¿Ha asistido a alguno de ellos? & $\square \mathrm{Si} \square \mathrm{No}$ \\
\hline $\begin{array}{l}\text { ¿Conoce usted el Plan de Iniciación a la Promoción Exterior } \\
\text { (PIPE)? }\end{array}$ & $\square \mathrm{Si} \square \mathrm{No}$ \\
\hline $\begin{array}{l}\text { ¿Conoce usted el Plan de Promotores de Comercio Exterior } \\
\text { elaborado por Excal? }\end{array}$ & $\square \mathrm{Si} \square \mathrm{No}$ \\
\hline ¿Conoce las iniciativas de Excal de apoyo a la exportación? & $\square \mathrm{Si} \square \mathrm{No}$ \\
\hline $\begin{array}{l}\text { ¿Conoce usted los centros de negocios de los que dispone Excal } \\
\text { en diferentes ciudades del mundo? }\end{array}$ & $\square \mathrm{Si} \square \mathrm{No}$ \\
\hline $\begin{array}{l}\text { ¿Conoce usted los consorcios de exportación entre empresas } \\
\text { que impulsa Excal? }\end{array}$ & $\square \mathrm{Si} \square \mathrm{No}$ \\
\hline ¿Ha acudido en alguna ocasión a solicitar los servicios de Excal? & $\square \mathrm{Si} \square \mathrm{No}$ \\
\hline $\begin{array}{l}\text { ¿Conoce usted el programa Castilla y León Exporta impulsado } \\
\text { por Excal? }\end{array}$ & $\square \mathrm{Si} \square \mathrm{No}$ \\
\hline $\begin{array}{l}\text { ¿Conoce usted si Pinacal/Aetical promueve la exportación de } \\
\text { las empresas asociadas? }\end{array}$ & $\square \mathrm{Si} \square \mathrm{No}$ \\
\hline $\begin{array}{l}\text { ¿Ha acudido en alguna ocasión a Pinacal/Aetical a solicitar } \\
\text { apoyo para la exportación de su empresa? }\end{array}$ & $\square \mathrm{Si} \square \mathrm{No}$ \\
\hline $\begin{array}{l}\text { ¿Ha consultado en alguna ocasión el portal del Icex? (http:// } \\
\text { www.icex.es) }\end{array}$ & $\square \mathrm{Si} \square \mathrm{No}$ \\
\hline
\end{tabular}

\section{Cuadro 1}

\section{Resultados}

En la tabla 1 se resumen los resultados obtenidos respecto a la valoración de atractivo, barreras y programas de ayuda en la internacionalización. También se recoge la valoración media que hacen los directivos de las diferentes fuentes de información en relación con los tres conceptos considerados.

A la vista de los resultados obtenidos llaman la atención dos cuestiones. En los dos sectores se comprueba que, de los tres aspectos considerados, la valoración directiva de los programas de ayuda es la que obtiene mayor puntuación y el atractivo el que obtiene la menor puntuación. Es decir, los directivos reconocen que en el proceso de internacionalización afecta decisivamente la información sobre apoyos y barreras mientras que la del atractivo es menos relevante. Por otro lado, la valoración que hacen los directivos del sector TIC resulta, en los tres aspectos, superior a la que indican los de las empresas de PN. Esa diferencia puede atribuirse a la distinta trayectoria de los dos sectores: el sector TIC cuenta con mayor recorrido que el sector $\mathrm{PN}$ en cuanto a la expansión internacional. Quizás por eso los directivos de las empresas TIC son más conscientes del valor de la información.

\section{"La valoración directiva de los programas de ayuda es la que obtiene la mayor puntuación, y el atractivo, la menor"}

Por lo que respecta a las fuentes de información, en primer lugar se

\begin{tabular}{|l|c|c|c|c|c|c|}
\hline & \multicolumn{3}{|c|}{ Empresas TI C } & \multicolumn{3}{c|}{ Empresas PN } \\
\hline FUENTES DE I NFORMACI ÓN & Atractivo & Barreras & Apoyos & Atractivo & Barreras & Apoyos \\
\hline Cámaras de comercio & 2,9 & 2,3 & 2,9 & 2,1 & 2,0 & 2,1 \\
Excal & 3,1 & 3,0 & 3,3 & 3,6 & 3,1 & 3,1 \\
Icex & 3,8 & 2,9 & 3,3 & 1,8 & 1,8 & 1,9 \\
Asociación sectorial & 3,8 & 2,7 & 2,6 & 3,6 & 2,7 & 2,9 \\
Promedio & $\mathbf{3 , 4}$ & $\mathbf{2 , 7}$ & $\mathbf{3 , 0}$ & $\mathbf{2 , 7}$ & $\mathbf{2 , 4}$ & $\mathbf{2 , 5}$ \\
\hline
\end{tabular}

Tabla 1. Valoración directiva del atractivo, barreras y apoyos, y evaluación de las diferentes fuentes de información 
constata la diversidad de fuentes de las que se nutren los directivos a la hora de valorar los distintos aspectos que intervienen en su decisión. No obstante, los datos obtenidos reflejan desequilibrios muy llamativos entre ellas.

\section{Fuentes de información en las empresas TIC}

En este sector el organismo regional de promoción de la internacionalización empresarial (Excal) constituye la referencia más relevante para la valoración de las barreras y los apoyos, seguido del correspondiente organismo nacional (Icex). En el caso de evaluación del atractivo las fuentes más citadas son el Icex y la asociación de empresarios del sector. Así, el Icex representa la más completa, puesto que resulta una de las más relevantes para los tres conceptos que intervienen en la decisión de internacionalización. La Cámara de Comercio proporciona estímulos informativos débiles en los tres aspectos considerados, sobre todo para las barreras. Excal constituye un referente en cuanto a las barreras y los programas de ayuda a la internacionalización empresarial. Por último, la asociación sectorial Aetical es clave a la hora de informarse sobre el atractivo y no tanto a la hora de valorar las barreras. Además, es el referente de menor importancia en lo que se refiere a apoyos y programas de ayuda. Respecto a la valoración global de estos organismos los datos indican que proporcionan mayoritariamente información sobre el atractivo, seguido de los programas de ayuda. La información que proporcionan sobre las barreras a la internacionalización es, sin embargo, la más escasa.

\section{Fuentes de información en las empresas PN}

En este sector los resultados son ligeramente distintos. En primer lugar observamos la relevancia de Excal para los tres conceptos considerados. La asociación secto- rial (Pinacal) destaca a la hora de proporcionar información sobre el atractivo y, en menor medida, sobre apoyos y barreras. En el extremo opuesto se sitúan la Cámara de Comercio y, sobre todo, el Icex como fuentes menos pertinentes en cuanto a su función de informantes. Por lo tanto, en este sector, los emisores que proporcionan más información se concentran, sobre todo, en la agencia regional de promoción de la exportación (Excal) y en la asociación sectorial (Pinacal). Se comprueba que, en conjunto, la información sobre el atractivo es la más abundante, seguida de la de apoyos. Tal y como sucedía en el sector TIC, la información sobre barreras es la que resulta más escasa.

Para completar el estudio examinamos la relación que hay entre la valoración de los factores determinantes de la decisión de internacionalización y dos elementos:

- la información recogida de las diversas fuentes

- las propiedades y actitudes cognitivas de los decisores empresariales.

Para ello hicimos un análisis de regresión en el que se considera la capacidad de explicación que muestran las variables cognitivas (nivel de proactividad y conocimiento) y la información de las diferentes fuentes respecto a la valoración que realizan los directivos de los elementos determinantes de la internacionalización (tabla 2).
Los resultados obtenidos revelan que la valoración que realizan los directivos de los factores determinantes de la internacionalización (atractivo, apoyos y barreras) viene explicada tanto por la información que reciben de diferentes fuentes como por sus propias propiedades cognitivas. En concreto se comprueba que:

- La valoración del atractivo depende de manera significativa de la actitud más o menos proactiva del directivo y no de la información recibida sobre el interés estratégico de la internacionalización.

- La valoración de las barreras depende de la información recibida de las distintas fuentes de información y del grado de conocimiento del directivo de los distintos foros e instrumentos relacionados con la internacionalización.

- La valoración directiva de los apoyos depende del grado de proactividad individual del directivo y de la información recibida sobre programas de ayuda a la expansión internacional de las empresas.

\section{Discusión e implicaciones prácticas}

El análisis de los factores que determinan la valoración de los distintos aspectos que intervienen en la internacionalización refleja la importancia de las propiedades cognitivas de los directivos. Se comprueba que la valoración del atractivo depende exclusivamente del nivel de proactividad del directivo y no de la información recibida.

\begin{tabular}{|l|l|l|l|}
\hline & Atractivo & Barreras & \multicolumn{1}{|c|}{ Apoyos } \\
\hline $\mathrm{R}^{2}$ & 0,32 & 0,22 & 0,435 \\
( $\mathrm{R}^{2}$ ajustado) & $(0,25)$ & $(0,14)$ & $(0,38)$ \\
\hline $\mathrm{F}$ (sig.) & $4,7(0,008)$ & $2,8(0,056)$ & $7,7(0,001)$ \\
\hline Constante & 0,80 & $4,77^{*} *$ & $1,27 *$ \\
Conocimiento & 0,67 & $-1,09 *$ & $-0,41$ \\
Proactividad & $2,08 * *$ & $-0,91$ & $2,03 *$ \\
Información atractivo & 0,27 & - & - \\
Información barreras & - & $0,25^{*}$ & - \\
Información apoyos & - & - & $0,336 * *$ \\
\hline
\end{tabular}

** variable significativa al $99 \%$ de confianza. * variable significativa al $90 \%$ de confianza. Tabla 2. Análisis de regresión de los factores determinantes de la internacionalización 
En conjunto, los resultados obtenidos reflejan la relevancia de las cuestiones vinculadas con el acceso a la información y cómo se combinan con las propiedades cognitivas de los decisores para conformar la valoración de los factores que convergen en la decisión de internacionalización. Además los resultados reflejan que los directivos consultados reciben más información sobre el atractivo que sobre barreras y apoyos, y esto a pesar de que esa información apenas afecta a la valoración directiva del atractivo. Simultáneamente los directivos manifiestan que la información referida a barreras y apoyos resulta más escasa.

Los organismos o instituciones que ayudan sobre distintos aspectos de la internacionalización empresarial deben analizar hasta qué punto su información contribuye a la valoración directiva de los elementos que determinan el proceso de internacionalización. Más aún, deben buscar una sintonía entre la naturaleza de la información que proporcionan y el papel que desempeña esa información.

\section{Agradecimientos}

Los autores agradecen el soporte financiero proporcionado por la Consejería de Educación de la Junta de Castilla y León (España) (Ref. VA038A08). También agradecen a la Asociación de Productores de Piedra Natural de Castilla y León (Pinacal) y a la Federación de Asociaciones de Empresas de Tecnologías de la Información, Comunicaciones y Electrónica de Castilla y León (Aetical) la ayuda recibida en el proceso de recogida de información. Agradecemos también a los evaluadores de EPI sus valiosos comentarios.

\section{Referencias bibliográficas}

Alonso, J. A. "El proceso de internacionalización de la empresa". Información comercial española, 1994, n. 725, enero, pp. 127-143.

Baron, R. A. "Cognitive mechanisms in entrepreneurship: why and when entrepreneurs think differently than other people". Journal of business venturing, 1998, v. 13, n. 4, pp. 275-294.

Burton, F. N.; Schlegelmilch, B. B. "Profile analyses of non-exporters versus exporters grouped by export involvement". Management international review, 1987 , v. 27 , n. 1, pp. 3849.

Calderón, H.; Fayos, T. "Análisis de la relación entre el compromiso exportador y las ayudas a la internacionalización de las empresas". Investigaciones europeas de dirección y economía de la empresa, 2004, v. 10, n. 2, pp. 201-220.

Carolis, D. M.; Saparito, P. "Social capital, cognition, and entrepreneurial opportunities: a theoretical framework". Entrepreneurship theory and practice, 2006, v. 30, n. 1, pp. 41-56.

Castilla y León Exporta. "Entrevista con Alberto Prados Sastre", (2007.a), n. 10, pp. 12-14.

Castilla y León Exporta. "Entrevista con Miguel Ángel García”, (2007.b), n. 11, pp. 12-14.

Claver, E.; Quer, D. "La dirección estratégica de la internacionalización de la empresa: propuesta de un marco teórico integrador". Información comercial española, 2001, n. 794, pp. 37-59.

Clarke, I.; Mackaness, W. "Management intuition: an interpretative account of structure and content of decision schemas using cognitive maps". Journal of management studies, 2001, v. 38 , n. 2, pp. 147-172.

Crant, M. J. "Proactive behaviour in organizations". Journal of management, 2000, v. 26, n. 3, pp. 435-462.

Dutton, J. E.; Ashford, S. J. "Selling issues to top management". Academy of Management review, 1993, v. 18, n. 3, pp. 397-428.

Dutton, J. E.; Ashford, S. J.; O’Neil, R. M.; Lawrence, K. A. "Moves the matter: issue selling and organizational change". Academy of Management journal, 2001, v. 44, pp. 716-736.

Gaglio, C. M.; Katz, J. A. "The psychological basis of opportunity identification: entrepreneurial alertness". Small business economics, 2001, v. 16, pp. 95-111.

Garg, V. K.; Walters, B. A.; Priem, R. L. "Chief executive scanning emphases, environmental dynamism, and manufacturing firm performance". Strategic management journal, 2003, v. 24, pp. 725-744.

Kaish, S.; Gilad, B. "Characteristics of opportunities search of entrepreneurs versus executives: sources, interests, general alertness". Journal of business venturing, 1991, v. 6, n. 1, pp. 45-61.

Katsikeas, C.; Morgan, R. "Differences in perception of exporting problems based on firm size and export marketing experience". European journal of marketing, 1994, v. 28, n. 15, pp. 17 33.

Leonidou, L. C. "Export stimulation research review, evaluation and integration", International business review, 1995, v. 4, n. 2, pp. 133-156.

Ling, Y.; Floyd, S. W.; Baldridge, D. C. "Toward a model of issue-selling by subsidiary managers in multinational organizations". Journal of international business studies, 2005, v. 36, pp. 637-654.

McEvely, B.; Peronne, V.; Zaheer, A. "Trust as an organizing principle". Organization science, 2003, v. 14, n. 1, pp. 91-103.

Manolova, T. S.; Brush, C. G.; Greene, P. G. "Internationalization on small firms". International small business journal, 2002, v. 20, n. 1, pp. 9-31.

Matlin, M. W. "Cognition", $5^{\text {th }}$ ed. Harcourt College, Fort Wort, TX, 2002.

Ozgen, E.; Baron, R. A. "Social sources of information in opportunity recognition: effects of mentors, industry networks, and professional forums". Journal of business venturing, 2007, v. 22, n. 2, pp. 174-192.

Ramírez, M.; Rosell, J. "La actividad internacional de la empresa aragonesa". Boletín económico de ICE, 2006, n. 2894, pp. 29-44.

Santos, M. V.; García, M. T. "The export decision and the cognitive limitation of the managers". Journal of information and knowledge management, 2008, v. 7, n. 2, pp. 123-133.

Shane, S. "Prior knowledge and discovery of entrepreneurial opportunities". Organization science, 2000, v. 11, pp. 448-469.

Shane, S. A general theory of entrepreneurship: the individual-opportunity nexus. Cheltenham: Edward Elgar, 2003.

Shane, S.; Venkataraman, S. "The promise of entrepreneurship as a field of research". Academy of Management review, 2003, v. 25, n. 1, pp. 217-226.

Weick, K. The social psychology of organizing. Reading: Addison-Wesley, 1979.

Wiedersheim-Paul, F.; Olson, H. C.; Welch, L. S. "Pre-export activity: the first step in internationalization". Working paper, Center of International Business Studies. Department of Business Administration, Uppsala University, 1978.

María-Valle Santos-Álvarez y María-Teresa García-Merino, Facultad de Ciencias Económicas y Empresariales, Universidad de Valladalid.

Avda. Valle Esgueva, 6. 47011 Valladolid, España.

mvalle@eco.uva.es

temerino@eco.uva.es 